\title{
A Compact Vivaldi Antenna Array for 5G Channel Sounding Applications
}

\author{
Naser Ojaroudi Parchin* and Raed Abd-Alhameed \\ Faculty of Engineering and Information, School of Electrical Engineering and Computer Science \\ University of Bradford, West Yorkshire, United Kingdom \\ *N.OjaroudiParchin@bradford.ac.uk
}

\begin{abstract}
A compact design of phased array Vivaldi antenna for channel sounding applications is proposed. 64elements of low-profile $22 \mathrm{GHz}$ Vivaldi antennas with coaxialto-SIW feeds and well-defined end-fire radiation patterns have been used to form an $8 \times 8$ planar array design. The antenna elements are designed on $1.6 \mathrm{~mm}$ Arlon AD-320 substrates $(\varepsilon=3.2$ and $\delta=0.0038)$. The structure of SIW is composed of two rows of cylinders between metal plates which can improve the radiation performance of the elements. The proposed phased array antenna has compact size with frequency response of 21-23 GHz. It has good features in terms of impedance-matching, gain and efficiency characteristics. More than $22 \mathrm{~dB}$ realized gain, $23 \mathrm{dBi}$ directivity, and $-0.5 \mathrm{~dB}(90 \%)$ total efficiency have been obtained. In addition, the performance of the planar array with different numbers of the radiators has been investigated.
\end{abstract}

Index Terms-5G, channel sounding, phased array design, Vivaldi antenna.

\section{INTRODUCTION}

Compared with the current and previous generations ( $1 \mathrm{G}$ to $4 \mathrm{G}$ ) of wireless communications, fifth generation (5G) networks are proposing to use the higher frequency bandwidths (beyond $10 \mathrm{GHz}$ ) due to growing need for wider bandwidths and higher data rates [1-2]. 22, 28, and $38 \mathrm{GHz}$ are the candidate bands for $5 \mathrm{G}$ wireless communications [3]. Our work represents a primarily study on the design of $22 \mathrm{GHz}$ Vivaldi antenna array for $5 \mathrm{G}$ channel sounding applications. The $5 \mathrm{G}$ phased array MIMO channel sounders are mainly based on an approach of switched beams. Using the phased arrays of antennas, the beams could be switched into different angles at both transmitter and the receiver [4].

Vivaldi antennas have received considerable amount of research effort owning to their attractive futures such as high gain, end-fire radiation mode, relatively wide band compared with typical antennas. The Vivaldi antennas could be used to form multiple smart-antenna systems for channel measurements [5-6].

The configuration of the employed Vivaldi antenna element is composed of two copper layer flares at both sides of the antenna substrate which is fed by the coaxial-toSIW technique. The designed antenna is operating in the frequency range of $21-23 \mathrm{GHz}$ and has high efficiency and sufficient gain values. The frequency response of the proposed design can be controlled by adjusting the feeding point of the Vivaldi antenna elements. Using the proposed
Vivaldi elements, the fundamental radiation characteristics of $2 \times 2,4 \times 4,1 \times 8$, and $8 \times 8$ arrays are studied.

\section{Phased ARRAy Vivaldi ANTENNA DESIGN}

Figure 1 illustrates the geometry of the proposed planar antenna. As illustrated, 64-elements of $22 \mathrm{GHz}$ Vivaldi antennas with Coaxial-to-SIW Feeds have been used for the proposed compact design. The radiation elements of the planar array are design on $1.6 \mathrm{~mm}$ Arlon AD-320 substrates to work at $22 \mathrm{GHz}$.

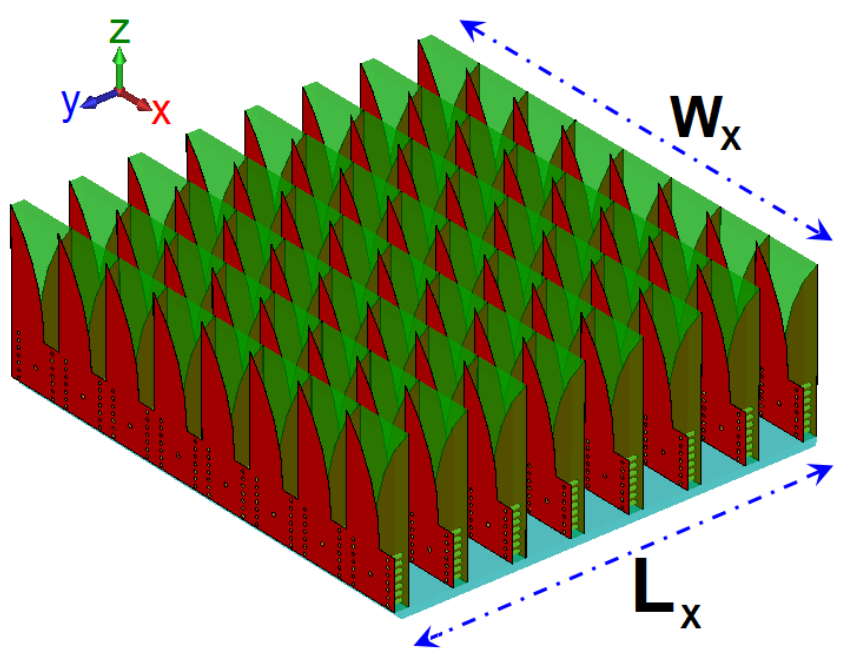

Fig. 1. Configuration of the proposed Vivaldi antenna phased array.

\section{Single ELEMENT VIVALDI ANTENNA}

The configuration of the single element $22 \mathrm{GHz}$ Vivaldi antenna is displayed in Fig. 2. As illustrated, it is composed of two copper layer flares at both sides of the antenna substrate which is fed by Coaxial-to-SIW technique. The antenna has a compact size with overall dimension of $\mathrm{W}_{\mathrm{S}} \times \mathrm{L}_{\mathrm{S}}$. The parameter values of the single element and the proposed array are specified in Table I.

Figure 3 illustrates the $S_{11}$ characteristic of the design Vivaldi antenna. As seen, the proposed Coaxial-to-SIW-fed Vivaldi antenna covers the frequency band of $21-23 \mathrm{GHz}$ and has a reflection coefficient of $-40 \mathrm{~dB}$. The frequency response of the designed Vivaldi can be controlled by adjusting the feeding point. 


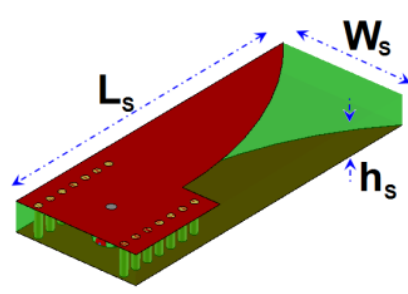

(a)

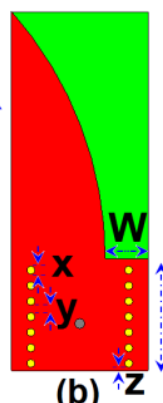

(b) ${ }^{\imath}$

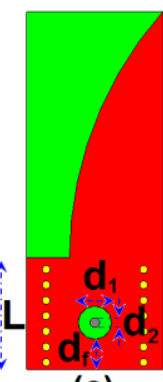

(c)
Fig. 2. (a) 3D-transparent, (b) top, and (c) bottom profiles of the antenna.

TABLE. I FINAL DIMESIONS OF THE VIVALDI ANTENNA PARAMETERS

\begin{tabular}{|c|c|c|c|c|c|}
\hline Parameter & $\mathrm{W}_{\mathrm{X}}$ & $\mathrm{Lx}$ & $\mathrm{W}_{\mathrm{s}}$ & $\mathrm{hs}$ & $\mathrm{W}$ \\
\hline Value $(\mathrm{mm})$ & 56 & 50.6 & 7 & 1.6 & 2.2 \\
\hline Parameter & $\mathrm{L}$ & $\mathrm{d}$ & $\mathrm{d}_{1}$ & $\mathrm{~d}_{2}$ & $\mathrm{~d}_{\mathrm{f}}$ \\
\hline Value $(\mathrm{mm})$ & 5.75 & 7 & 1.72 & 0.5 & 1.56 \\
\hline Parameter & $\mathrm{W}_{\mathrm{a}}$ & $\mathrm{L}_{\mathrm{a}}=\mathrm{Ls}_{\mathrm{s}}$ & $\mathrm{x}$ & $\mathrm{y}$ & $\mathrm{z}$ \\
\hline Value $(\mathrm{mm})$ & 56 & 18.5 & 0.4 & 0.4 & 0.175 \\
\hline
\end{tabular}

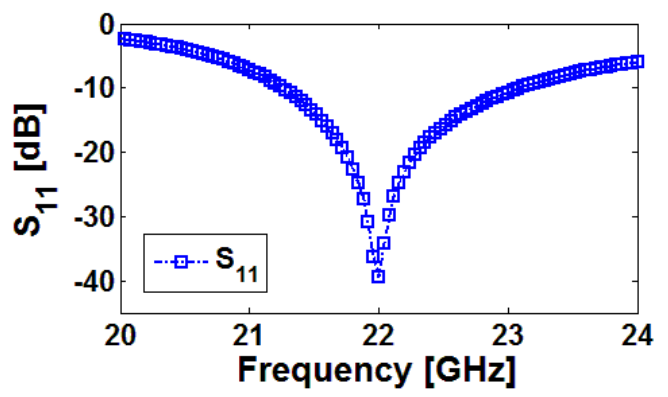

Fig. 3. $\mathrm{S}_{11}$ characteristic of the designed Vivaldi antenna.

The 3D and radiation pattern of the antenna at $22 \mathrm{GHz}$ is illustrated in Fig. 4 (a). As seen, the antenna has a desirable end-fire radiation property with $4.46 \mathrm{~dB}$ realized gain (IEEE gain $\times$ missmatch). In order to demonstrate that the antenna actually radiates over the operation frequency band ( 21 to $23 \mathrm{GHz}$ ), its fundamental properties are illustrated in Fig. 4 (b). Based on the obtained results, the antenna has high radiation and total efficiencies with more than $4 \mathrm{dBi}$ maximum gain.

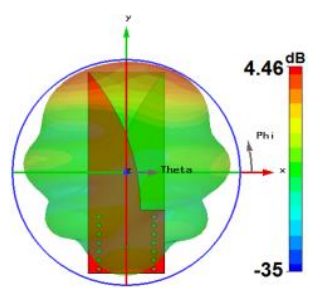

(a)

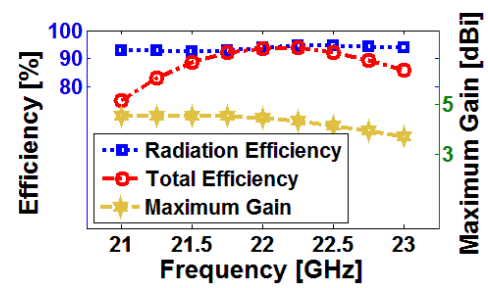

(b)
Fig. 4. Simulated (a) antenna radiation pattern at $22 \mathrm{GHz}$, and (b) fundamental properties of the antenna over the operation band.

\section{RADIATION PERFORAMNCE OF THE ARRAY DESIGN}

The proposed phased array antenna is designed using eight rows of $1 \times 8$ linear arrays. In this section, radiation and input-impedance characteristics of the linear Vivaldi antenna array has been investigated firstly. Configuration of the linear array with eight Vivaldi antenna elements is shown in Fig. 5.

Figure 6 shows the $S$ parameters $\left(S_{11}\right.$ to $\left.S_{81}\right)$ of the array. As shown, the array has a good performance in the frequency range of 21 to $23 \mathrm{GHz}$. The highest mutualcoupling characteristic $\left(S_{21}\right)$ between the Vivaldi antenna elements is less than $-19 \mathrm{~dB}$ at the center frequency (22 $\mathrm{GHz}$ ).

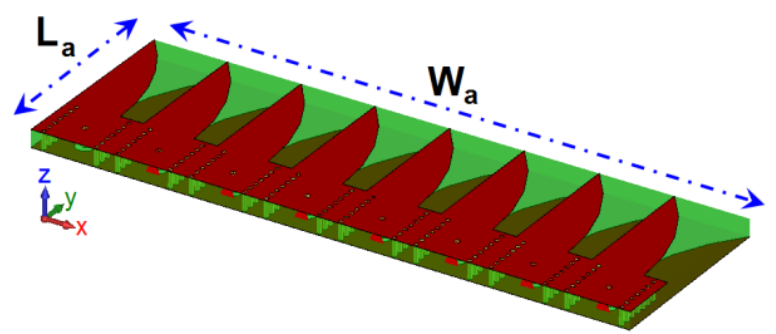

Fig. 5. Configuration of the Vivaldi antenna array with eight radiators.

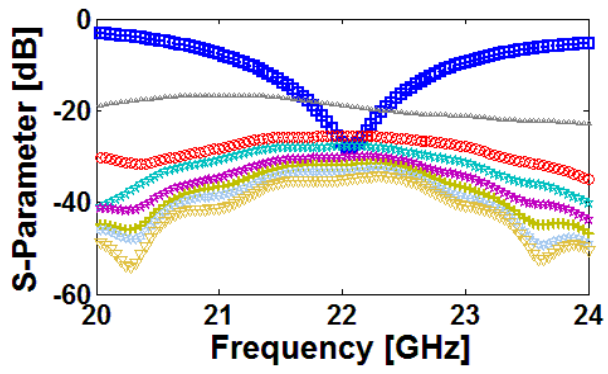

Fig. 6. S parameters of the array shown in Fig. 5.

$3 \mathrm{D}$ radiation beams of the linear Vivaldi antenna array with directivity values at $0,30,45$ and 60 degrees are shown in Fig. 7 (a). The designed array has good beam steering characteristic with wide range beam-coverage property. Furthermore, the simulated realized gains (Cartesian) of the array at different scanning angles are represented in Fig. 7 (b). It can be seen that the array has sufficient gain levels at different scanning angles.

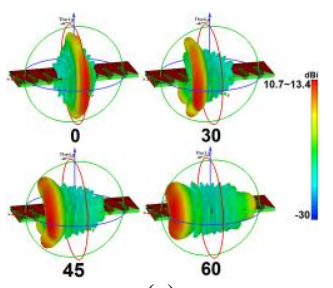

(a)

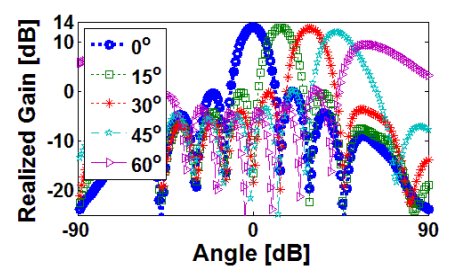

(b)
Fig. 7. (a) $3 \mathrm{D}$ and (b) $2 \mathrm{D}$ radiation beams of the arrays at different angles.

64 elements of the Vivaldi antennas have been used to design the final structure (Fig. 1). The 3D directional radiation beams of the proposed planar Vivaldi antenna with realized gain values at different angles are represented in Fig 8. It can be seen that the proposed antenna has good beam steering characteristic with high-level realized gains at the different scanning angles. 


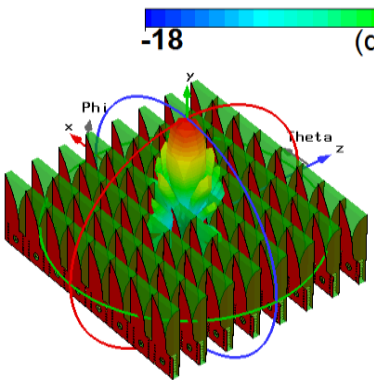

(a)

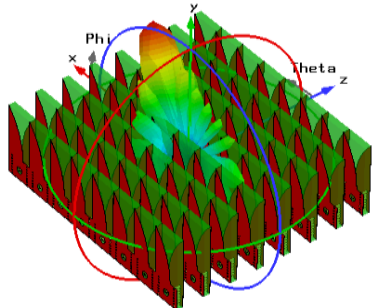

(c)

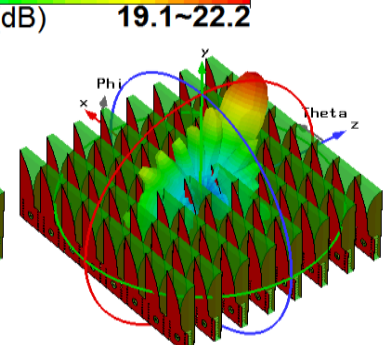

(b)

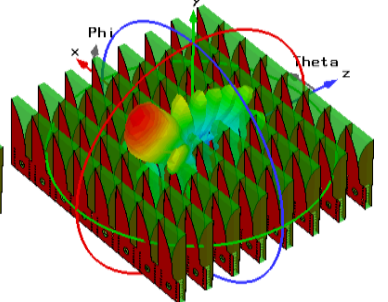

(d)
Fig. 8. 3D radiation patterns of the array at different scanning angles

\section{INVESTIGATION ON THE PERFORMANCES OF THE $2 \times 2,4 \times 4$, AND $8 \times 8$ PLANAR ARRAYS}

The radiation and input-impedance characteristics of the proposed $22 \mathrm{GHz}$ planar Vivaldi antenna array with $2 \times 2,4 \times 4$, and $8 \times 8$ numbers of the antenna elements have been investigated in this section. The antenna elements are arranged with the distance of $\mathrm{d}=7 \mathrm{~mm}$. Reflection coefficient $\left(S_{11}\right)$ and mutual coupling $\left(S_{21}\right)$ characteristics of the planar arrays are illustrated in Fig. 9.

Sufficient and almost similar performances of the frequency response have been achieved for the arrays. As seen in Fig. 9 (a), $-27,-28$, and $-25 \mathrm{~dB}$ reflection coefficients are obtained for the $2 \times 2,4 \times 4$, and $8 \times 8$ planar arrays. In addition, as illustrated in Fig. 9 (b), the designed arrays have low mutual coupling between radiators (less than $-17 \mathrm{~dB})$.

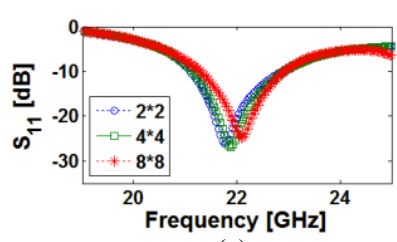

(a)

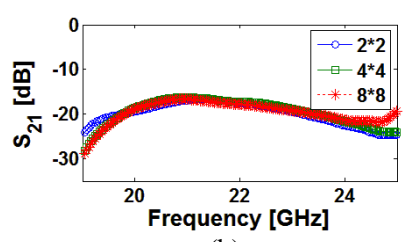

(b)
Fig. 9. Simulated (a) $S_{11}$ and (b) $S_{21}$ characteristics of the designed arrays.

$3 \mathrm{D}$ radiation beams of the arrays when their beams are tilted to $0^{\circ}$ elevation are shown in Fig. 10. More than 10.5, 16.5 , and $23 \mathrm{dBi}$ directivity values with good radiation behaviors and low back lobes have been achieved for the planar arrays. Table II summarizes the performances of the designed arrays in terms of different antenna parameters. As seen, the arrays exhibit good radiation and inputimpedance characteristics.

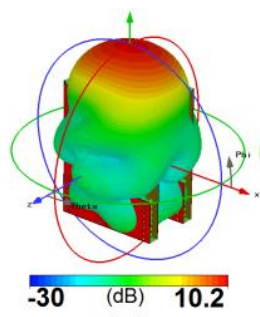

(a)

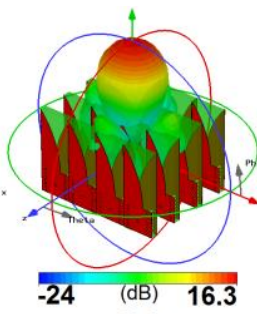

(b)

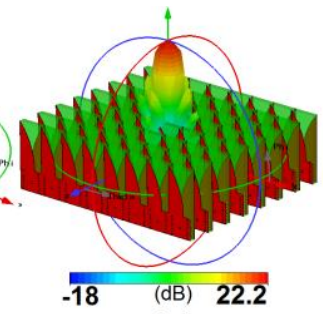

(c)
Fig. 10. The beams at 0 degree for (a) $2 \times 2$, (b) $4 \times 4$, and (c) $8 \times 8$ arrays.

TABLE. II FINAL DIMESIONS OF THE 5G ANTENNA PARAMETERS

\begin{tabular}{|c|c|c|c|c|c|}
\hline Array/Param. & Gain & Effic. & BW & R.C & M.C \\
\hline $\mathbf{1 \times 1}$ & $4.46 \mathrm{~dB}$ & $-0.4 \mathrm{~dB}$ & $2 \mathrm{GHz}$ & $-40 \mathrm{~dB}$ & -- \\
\hline $\mathbf{2} \times \mathbf{2}$ & $10.2 \mathrm{~dB}$ & $-0.39 \mathrm{~dB}$ & $1.9 \mathrm{GHz}$ & $-25 \mathrm{~dB}$ & $-17 \mathrm{~dB}$ \\
\hline $\mathbf{4} \times \mathbf{4}$ & $16.3 \mathrm{~dB}$ & $-0.47 \mathrm{~dB}$ & $1.8 \mathrm{GHz}$ & $-25 \mathrm{~dB}$ & $-17 \mathrm{~dB}$ \\
\hline $\mathbf{8} \times \mathbf{8}$ & $22.2 \mathrm{~dB}$ & $-0.5 \mathrm{~dB}$ & $1.8 \mathrm{GHz}$ & $-25 \mathrm{~dB}$ & $-18 \mathrm{~dB}$ \\
\hline
\end{tabular}

\section{CONCLUSION}

A compact planar phased array antenna for $5 \mathrm{G}$ channel sounding applications is proposed in this manuscript. Simulations including $\mathrm{S}$ parameter, efficiency, radiation pattern characteristics are investigated and good results have been obtained. The proposed planar array antenna is formed by using 64-elements of $22 \mathrm{GHz}$ Vivaldi antennas. The antenna provides some attractive features such as high gain, high efficiency, small size and could be used in the future wireless systems.

\section{ACKNOWLEDGMENT}

This work is supported by the European Union's Horizon 2020 research and innovation programme under grant agreement H2020-MSCA-ITN-2016 SECRET722424.

\section{REFERENCES}

[1] P. Gupta, "Evolvement of mobile generations: $1 \mathrm{G}$ to 5G," International Journal for Technological Research in Engineering, vol. 1, pp. 152-157, 2013.

[2] A. Osseiran, et al., "Scenarios for 5G mobile and wireless communications: the vision of the METIS project," IEEE Commun. Mag., vol. 52, pp. 26-35, 2014.

[3] W. Roh et al., "Millimeter-wave beamforming as an enabling technology for 5G cellular communications: Theoretical feasibility and prototype results,“ IEEE Commun. Mag., vol. 52, pp. 106-113, 2014.

[4] Z. Pi and F. Khan, "A millimeter-wave massive MIMO system for next generation mobile broadband", ASILOMAR 2012, Pacific Grove, CA, USA, pp. 693-698.

[5] P. Xingdong H. Wei ; Y. Tianyang; L. Linsheng, "Design and implementation of an active multibeam antenna system with $64 \mathrm{RF}$ channels and 256 antenna elements for massive MIMO application in $5 \mathrm{G}$ wireless communications," Communications, China, vol. 11 , pp. $16-23,2014$.

[6] P. Fei, X. Wen, P. Zhang, Y.-C. Jiao "A broad band \#-shaped 4element tapered slot antenna array for measurement applications Wireless Symposium (IWS), 2015 IEEE International, Shenzhen, March 2015. 